\title{
Interferometric characterization of 160 fs far-infrared light pulses
}

\author{
B. I. Greene, J. F. Federici, and D. R. Dykaar \\ AT\& $T$ Bell Laboratories, 600 Mountain Avenue, Murray Hill, New Jersey 07974
}

R. R. Jones and P. H. Bucksbaum

Department of Physics, University of Michigan, Ann Arbor, Michigan 48109

(Received 18 March 1991; accepted for publication 28 May 1991)

\begin{abstract}
We report the first interferometric characterization of freely propagating, subpicosecond, far-infrared (FIR) light pulses. FIR light was generated via short pulse photoexcitation of a semi-insulating InP wafer. The half width of the intensity interferogram was $230 \mathrm{fs}$. The FIR light contained frequency components from 3 to $150 \mathrm{~cm}^{-1}$.
\end{abstract}

Several schemes of far-infrared (FIR) light emission based on either picosecond time scale current surges ${ }^{1-6}$ or on Cerenkov radiation ${ }^{7}$ in electro-optlc crystals have been presented. Preliminary attempts at addressing questions regarding the mechanisms of FIR light pulse generation have been hampered by the lack of absolute information on the spectral content and temporal duration of these pulses. This situation exists primarily due to the present techniques of pulsed FIR light detection.

Specifically focusing on work with freely propagating FIR light pulses, it has proven convenient to utilize antenna structures to efficiently gather incident electromagnetic radiation. The feedpoint of these antennas is bridged by a fast photoconductive material, capable of "gating" the electromagnetic energy off of the structure with roughly $0.5 \mathrm{ps}$ resolution. The gate duration puts an effective upper limit on the detection bandwidth at roughly $70 \mathrm{~cm}^{-1}(\sim 2$ $\mathrm{THz}$ ). In principle, electro-optic sampling in a material such as $\mathrm{LiTaO}_{3}$ could provide much higher bandwidths. However, low generated FIR powers mandating extremely tight focusing in addition to the dispersive nature of the electro-optic media itself present serious technical obstacles for use with freely propagating light.

In this letter, we report the results of an interferometric study of FIR light pulses which is free of the bandwidth limitations discussed above. We attribute the success of this technique to the use of an amplified low-repetition rate visible pulsed laser system to generate the FIR light. Resultant FIR light pulses are easily detected with a conventional bolometer, alleviating the necessity of gated antennas or electro-optic detection.

A $10 \mathrm{~Hz}$ YAG pumped, amplified colliding-pulse, mode-locked (CPM) dye laser system was used to generate approximatcly $2 \times 10^{-4} \mathrm{~J}, 100 \mathrm{fs}$ light pulses at $630 \mathrm{~nm}$. The visible light excited a $\langle 100\rangle$ semi-insulating $\mathrm{Fe}$-doped InP wafer, generating a linearly polarized FIR light pulse. This light was collimated with an off-axis paraboloid and directed into a polarizing Michelson interferometer. ${ }^{8,9}$

The interferometer consisted of a free-standing wire grid polarizing beam-splitter ( $12 \mu \mathrm{m}$ spacing) rotated $45^{\circ}$ relative to the polarization of the incident FIR pulse. The reflected and transmitted FIR light pulses are of equal intensity and orthogonally polarized. Wire grid bcamsplitters of this wire spacing have been demonstrated to be spectrally flat out past $300 \mathrm{~cm}^{-1}$. Rooftop retroreflectors in each arm rotate the polarization of the incident light $90^{\circ}$. The orthogonal transmitted and reflected pulses are recombined at the beamsplitter. A final wiregrid polarizer (analyzer) transmits components of the combined outgoing beams with the same polarization. The inteferogram of the FIR light is recorded by varying the path length of one arm of the interferometer with respect to the other. The interferometer output was focused with another off-axis paraboloid into a helium-cooled silicon composite bolometer. The bolometer had a 1-mm-thick wedged polyethylene window and a 0.1 mil polyethylene $500 \mathrm{~cm}^{-1}$ low pass cold filter.

We report first on the energy characteristics of the FIR pulse. With an incident visible laser pulse of $2 \times 10^{-4} \mathrm{~J}$ and a spot size on the InP of roughly $2 \mathrm{~cm}^{2}$, an $8 \mathrm{~V}$ signal was observed after efficiently collecting the FIR light and directing it onto the bolometer. For a detector gain of $5.27 \times 10^{7} \mathrm{~V} / \mathrm{W}$ and a bolometer response time of $0.5 \mathrm{~ms}$, we estimate the total per pulse FIR energy to be $8 \times 10^{-11}$ J. Assuming an $\sim 200 \mathrm{fs}$ pulse width (see below), the peak power is on the order of $400 \mathrm{~W}$. The conversion efficiency from visible to FIR was $\sim 4 \times 10^{-7}$. The bolometer noise at this gain was $\sim 2 \mathrm{mV}$ indicating a signal to noise ratio greater than 1000 . The noise in the data presented below is therefore almost entirely laser (versus detector) generated.

Attempts to measure the FIR light generated from a InP crystal illuminated with the unamplified, unfocused output of a CPM laser failed. A lock-in amplifier and optical chopper were utilized, and signals on the order of 100 $\mu \mathrm{V}$ would have been detectable. It has been established that the scaling of FIR light with incident visible light has two regimes. ${ }^{5}$ At high visible pulse fluence, $10^{-6}-10^{-3}$ $\mathrm{J} / \mathrm{cm}^{2}$, a linear relationship between the visible intensity $I_{\mathrm{vis}}$ and the FIR intensity $I_{\mathrm{FIR}}$ is manifest. At significantly lower pulse fluence, $\sim 10^{-8} \mathrm{~J} / \mathrm{cm}^{2}, I_{\mathrm{FIR}}$ has been observed to vary quadratically with $I_{\text {vis, }}$, i.e., $I_{\text {FIR }} \propto I_{\text {vis }}^{2}$. Depending on exactly where the break point between quadratic and linear behavior occurs (our guess is between $10^{-7}$ and $10^{-6} \mathrm{~J} / \mathrm{cm}^{2}$ ), our amplified pulse results are consistent with a border line undetectable (with a bolometer) amount of FIR light generated by an unamplified unfocused CPM laser beam incident on InP.

A typical interferogram \{autocorrelation of the generated FIR light with constant offset subtracted $\left.S(\tau)=\int_{-\infty}^{\infty}[E(t)+E(t-\tau)]^{2} d t-2 \int_{-\infty}^{\infty} E^{2}(t) d t\right\}$ is 


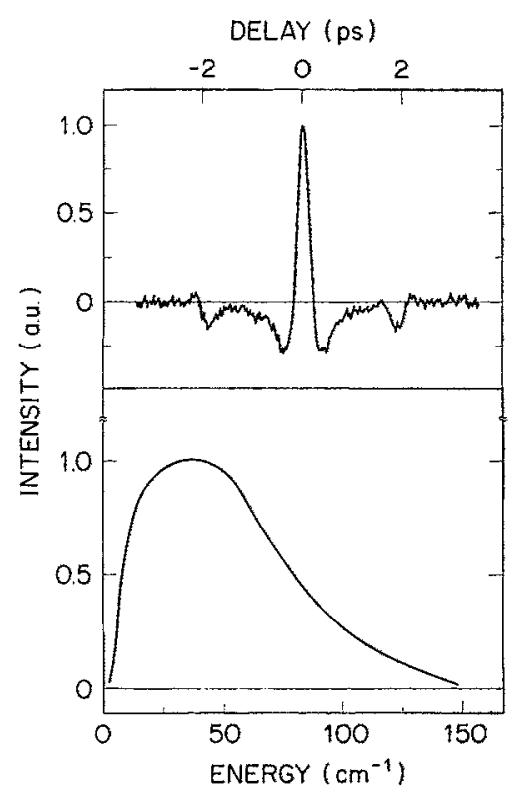

FIG. 1. (top) Interferogram of far-infrared light emitted from InP wafer. A constant offset has been subtracted as described in the text. (bottom) Fourier transform of interferogram. Satellite pulses at \pm 2 ps are mathematically removed prior to transform.

shown in the top half of Fig. 1. The width of the central peak is roughly $230 \mathrm{fs}$. The two satellite peaks at roughly \pm 2.0 ps are artifacts due to the bolometer. In detail, the composite sensing element consists of a 0.1 -nm-thick, 2.5 $\mathrm{mm}$ by $2.5 \mathrm{~mm}$ diamond substrate, the back of which has been blackened and a few micron sized Si bolometric element bonded. The expected round trip time for a FIR pulse of light through the diamond substrate is very close to the $\sim 2$ ps spacing observed in the interferogram. By simply wedging the substrate, we hope to remove this étalon effect in the future. The Fourier transform of a typical interfero- gram is shown in the bottom half of Fig. 1. The satellite pulses have been mathematically removed thus avoiding Fabry-Perot peaks associated with the diamond

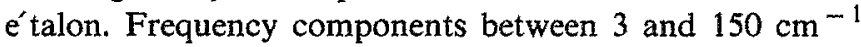
are clcarly evident.

Without an assumption of a specific functional form for the electric field, a unique transformation from the intensity autocorrelation to the parent pulse cannot be made. However, the simple assumption of a symmetric form for $E(t)$ allows one to obtain $E(t)$ from the intensity autocorrelation. Figure 2 (top) shows a typical autocorrelation for the FIR pulse, while Fig. 2 (bottom) was obtained by taking the inverse transform of the square root of the autocorrelation transform to yield $E(t)$.

The spatial properties of the FIR light were investigated as the light propagated off of the InP crystal. We observed that by distorting the optical wavefront the peak in the FIR intensity could be shifted as much as $\pm 3^{\circ}$ off the reflected visible light path. Undistorted optical beams produce FIR beams which were collinear with the reflected visible pulse, as previously reported ${ }^{3,4}$

Finally, we wish to comment on the apparent miscon-

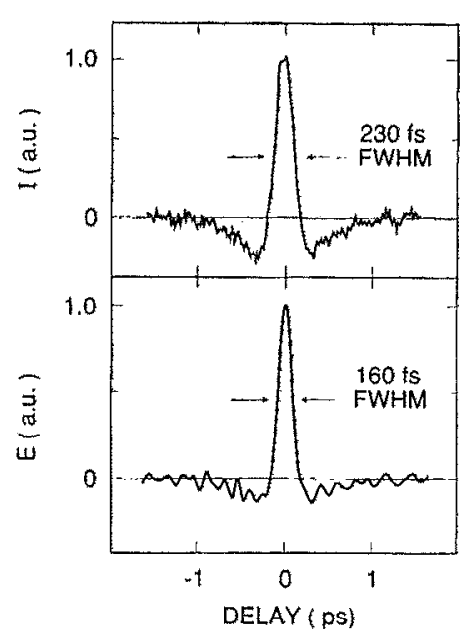

FIG. 2. (top) Interferogram of far-infrared light emitted from InP wafer. A constant offset has been subtracted as described in the text. (bottom) Inferred symmetric electric field $E(t)$ derived from top.

ception that by detecting FIR intensity instead of electric field, one loses the ability to directly (i.e., without resorting to Kramers-Kronig transformation) obtain phase information about a spectroscopic sample. Interferometers are ideally suited to measuring phase retardations. By placing a sample in one arm of a Michelson interferometer, one obtains an "asymmetric interferogram." Subsequent application of a complex inverse Fourier transform yields the requisite phase information. This technique has been extensively discussed and demonstrated in the literature. ${ }^{10,11}$

In conclusion, we have made the first absolute spectral characterization of freely propagating subpicosecond FIR light emitted from a photoexcited InP wafer. The measured frequency width of $3-150 \mathrm{~cm}^{-1}$ is the highest measured bandwidth of freely propagating subpicosecond FIR radiation to date. ${ }^{12}$ Additionally, the inferred electric field pulse width of $160 \mathrm{fs}$ is shorter by a factor of 2 than the shortest previously reported freely propagating FIR pulse. ${ }^{6}$ The interferometric detection scheme discussed in this letter demonstrates increased bandwidth and versatility over current gatable antenna detection techniques for both linear and nonlinear spectroscopic measurements.

The authors wish to acknowledge G. A. Thomas, D. H. Rapkine, S. Schmitt-Rink, and X.-C. Zhang for many valuable discussions. We are also grateful to $P$. C. Becker and A. G. Prosser for use of a high repetition rate amplified CPM system.

'P. R. Smith, D. H. Auston, and M. C. Nuss, IEEE J. Quantum Electron. 24, 255 (1988).

${ }^{2}$ M. van Exter, C. Fattinger, and D. Grischkowsky, Appl. Phys. Lett. 55, 337 (1989).

${ }^{3}$ X. C. Zhang, B. B. Hu, J. T. Darrow, and D. H. Auston, Appl. Phys. Lett. 56, 1011 (1990).

${ }^{4}$ J. T. Darrow, B. B. Hu, X.-C. Zhang, and D. H. Auston, Opt. Lett. 15, 323 (1990).

${ }^{5}$ B. I. Greene, J. F. Federici, D. R. Dykaar, A. F. J. Levi, and L. Pfeiffer, Opt. Lett. 16, 48 (1991). 
${ }^{6}$ N. Katzenellenbogen and D. Grischkowsky, Appl. Phys. Lett. 58, 222 (1991).

${ }^{7}$ D. H. Auston, K. P. Cheung, J. A. Valdmanis, and D. A. Kleinman, Phys. Rev. Lett. 53, 1555 (1984).

${ }^{8}$ W. G. Chambers, T. J. Parker, and A. E. Costley, Infrared and Millimeter Waves, (Academic, New York, 1985), Vol. 16, Chap. 3.

${ }^{9}$ C. H. Martin and E. Puplett, Infrared Phys. 10, 105 (1969).
${ }^{10}$ J. E. Chamberlain, J. E. Gibbs, and H. A. Gebbie, Infrared Phys. 9, 185 (1969).

${ }^{11}$ R. J. Bell, Introduction to Fourier Transform Spectroscopy (Academic, New York, 1972), p. 78.

${ }^{12}$ Note added in proof: using InP (111), we have recently observed $120 \mathrm{fs}$ FIR pulses with detectable light beyond $200 \mathrm{~cm}^{-1}$. 